\section{Ein Ionen-Laser für metrologische Anwendungen}

\section{E. ENgelhard und F. SPIEweck}

Mitteilung aus der Physikalisch-Technischen Bundesanstalt Braunschweig

(Z. Naturforsch. 25 a, 156 [1970] ; eingeg. am 17. Dezember 1969)

A xenon isotop laser has been built which emits $\mathrm{Xe}$ (II) lines in the visible. The vapour pressure controlled laser tube operates at low current. Preliminary vacuum wavelength values have been found to be $(542.06549 \pm 0.00002) \mathrm{nm}$ and $(627.25464 \pm 0.00001) \mathrm{nm}$.

Für metrologische Anwendungen wurde ein Laser entwickelt, der mehrere Linien im sichtbaren Spektralbereich emittiert. Das Laser-Rohr ist gefüllt mit dem Nuklid ${ }^{136} \mathrm{Xe}$ von einigen Torr Druck. Ein Zweig des Laser-Rohrs wird in siedendem Sauerstoff gekühlt, so daß sich der Sättigungsdruck des Xenons von etwa 45 mTorr einstellt. Daher sind die internen Bedingungen des Laser-Rohrs und somit die Wellenlängen stabil. Die wassergekühlte Kapillare aus Solidex-Glas hat einen Durchmesser von nur etwa $1 \mathrm{~mm}$, so daß der Entladungsstrom und damit die Einflüsse von Starkund Zeeman-Effekt klein bleiben. Zwischen dem Anoden- und dem Kathodenteil, die eine zylindrische Anode bzw. eine Glühkathode enthalten, befindet sich eine Gasrückführleitung ${ }^{1}$ (s. Abb. 1).

Bei Verwendung breitbandiger dielektrischer LaserSpiegel wurde die Emission einer roten $\left(6 \mathrm{p}^{\prime}{ }^{2} \mathrm{~F}_{5 / 2}^{0}-\right.$ $\left.6 \mathrm{~s}^{\prime}{ }^{2} \mathrm{D}_{3 / 2}, \lambda=627 \mathrm{~nm}\right)$ und einer grünen $\left(6 \mathrm{p}^{4} \mathrm{D}^{0}{ }_{5 / 2}-\right.$ $6 \mathrm{~s}^{4} \mathrm{P}_{3 / 2}, \lambda=542 \mathrm{~nm}$ ) Xe(II) -Linie ${ }^{2,3}$ beobachtet. Je nach Stärke des Entladungsstromes erscheinen bei einem Spiegelabstand von $43 \mathrm{~cm}$ ein bis fünf ${ }^{4}$ axiale Eigenschwingungen bei konfokaler Anordnung der Spiegel. Die Wellenlängen wurden zunächst im Betrieb mit drei Eigenschwingungen gemessen. Hierzu wurde der Abstand der Laser-Spiegel piezoelektrisch so eingestellt, daß die beiden äußeren mit Hilfe eines FabryPerot-Etalons feststellbaren Eigenschwingungen dem Auge gleich hell erschienen. Die Sichtbarkeit der Interferenzen erwies sich bei einem Gangunterschied von $1 \mathrm{~m}$ als völlig unbeeinträchtigt. Die an verschiedenen

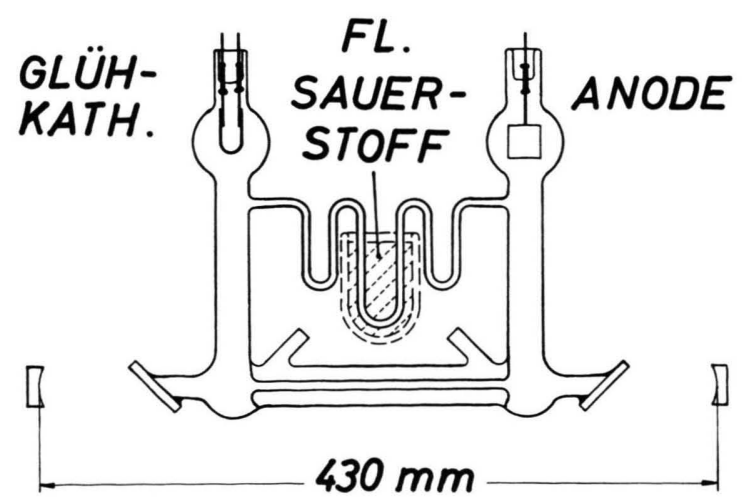

Abb. 1. Xenon-Ionen-Laser, schematisch.

Laser-Rohren und bei unterschiedlichen Entladungsstromstärken (1,3 A bis 2,2 A) ermittelten Wellenlängenwerte betragen $(542,06549 \pm 0,00002) \mathrm{nm}$ und $(627,25464 \pm 0,00001) \mathrm{nm}$ (Unsicherheitsangaben: einfache Standardabweichung).

Eine Abhängigkeit der Wellenlängen von Druck und Stromstärke konnte nicht festgestellt werden ${ }^{5}$. Angestrebt wird der Bau eines Einfrequenz-Lasers mit einer Emission bei den drei Wellenlängen $460 \mathrm{~nm}, 542 \mathrm{~nm}$ und $627 \mathrm{~nm}$. Dies wäre wichtig für die metrologische Anwendung der Koinzidenzmethode zur Bestimmung der Ordnungszahl von Interferenzen.

Unseren Mitarbeitern, den Herren WÜRTELE, DARnedde und WesselHÖFT, sind wir zu großem Dank verpflichtet für die Herstellung der Laser-Spiegel und für die sorgfältigen Messungen der Wellenlängen. Herr O. SchmidT hat sich großes Verdienst erworben bei der Lösung von schwierigen glastechnischen Aufgaben.

1 E. I. Gordon u. E. F. Labuda, Bell Syst. Tech. J. 43, 1827 [1964].

2 W. B. BRIDges, Proc. IEEE 52, 843 [1964].

3 E. F. Labuda u. A. M. Johnson, IEEE J. Quant. Electron. QE-2, 700 [1966].

4 A. L. Bloom, R. L. Byer u. W. E. Bell, Phys. Quant. Electron. (Confer. Proc.) Ed. P. L. Kelley, B. Lax u. P. E. TANNENwald, McGraw-Hill, 688 [1966].

5 Der Druck wurde bisher nur bei den Wellenlängenbestimmungen der roten Linie variiert. 